\title{
Le progrès, le transfert et le choix technologiques dans les pays en voie de développement (PVD) : vers une approche plus réaliste du problème de la substitution Technical progress, technology transfer and the choice of technique in the developing countries: towards a more realistic approach to the problem of substitution
}

\section{Eckhard Siggel}

Volume 58, numéro 3, juillet-septembre 1982

Le progrès technologique

URI : https://id.erudit.org/iderudit/601025ar

DOI : https://doi.org/10.7202/601025ar

Aller au sommaire du numéro

Éditeur(s)

HEC Montréal

ISSN

0001-771X (imprimé)

1710-3991 (numérique)

Découvrir la revue

Citer cet article

Siggel, E. (1982). Le progrès, le transfert et le choix technologiques dans les pays en voie de développement (PVD) : vers une approche plus réaliste du problème de la substitution. L'Actualité économique, 58(3), 303-322.

https://doi.org/10.7202/601025ar

\section{Résumé de l'article}

In this paper it is argued that the conventional estimation of production functions may be misleading for the study of technological progress and technology choice in developing countries. The analysis of technical progress and technology transfers requires empirical production functions which should reflect accurately the state of technology and productivity in a given country or region. The neoclassical production function embracing as an envelope all observations in an industry is likely to overstate the number of techniques already established in the region. It may therefore underestimate the technical progress achieved by further transfers. The problem lies in the very concept of technological progress which is defined as a shift of the universal production function and excludes movements along the production isoquant.

As to the choice of technology the estimation of the elasticity of substitution may be equally misleading for the purpose of predicting changes of factor use caused by factor price changes. The substitution possibilites between factors of production in the actually existing choice set of techniques for a given country or region are better described by the concept of a technology shelf. Two important characteristics of the technology shelf are the range and density of substitution. It is argued that industrial engineering information should be used to better describe the technology shelf. In its empirical part, using data of the manufacturing sector of Zaire, the paper shows how such engineering information may be used to estimate the range and density of substitution. 


\section{LE PROGRÈS, LE TRANSFERT ET LE CHOIX TECHNOLOGIQUES DANS LES PAYS EN VOIE DE DÉVELOPPEMENT (PVD) : VERS UNE APPROCHE PLUS RÉALISTE DU PROBLÈME DE LA SUBSTITUTION*}

Le problème des possibilités de substitution entre facteurs et celui du choix technologique occupent une place importante dans la littérature économique. La majorité des études abordent le problème comme une simple question empirique. Les "optimistes de l'élasticité" présentent des preuves supportant l'hypothèse de flexibilité; les "pessimistes de l'élasticité " quant à eux soutiennent que le choix technologique est plutôt limité. Dans la présente étude il ne s'agit pas de supporter principalement l'une ou l'autre de ces hypothèses. Notre approche a plutôt un but méthodologique. Il s'agit d'examiner les hypothèses explicites et implicites sur lesquelles se basent les études du progrès, du transfert et du choix technologiques en utilisant des fonctions de production. Le refus de quelques-unes de ces hypothèses remet en question l'utilité de la fonction de production néoclassique pour certaines fins. Dans la première section, les faiblesses du concept économique du progrès technologique sont attribuées à la définition de la fonction de production. Dans les sections deux et trois, les cas du progrès et transfert technologiques et du choix technologique sont analysés successivement. Dans les sections 4 et 5 , quelques méthodes alternatives pour évaluer les possibilités de substitution sont explorées à l'aide de données recueillies au Zaïre.

\section{I- LES FAIBLESSES DU CONCEPT ÉCONOMIQUE DE PROGRÈS TECHNOLOGIQUE}

Le concept du progrès technologique tel qu'utilisé dans la littérature économique est celui d'un déplacement de la fonction de production. Celle-ci reflète toutes les combinaisons des facteurs de production et des maximums de production possibles étant donné le niveau actuel de connaissances scientifiques et techniques. Comme il a été souligné par Rosenberg ce concept n'est pas approprié pour analyser l'origine et les

* Je tiens à remercier mes anciens professeurs G.K. Helleiner, A. Berry et T. Rawski, mon collègue $P$. Callier et deux lecteurs anonymes de cette revue pour des discussions et suggestions utiles. 
causes du progrès technologique ${ }^{1}$. Nous argumenterons ici que le concept est aussi peu approprié pour analyser le progrès technologique dans les pays en voie de développement (PVD).

Le progrès technologique dans les PVD se distingue de celui des pays industrialisés en ce qu'il se présente habituellement comme substitution des techniques désuètes par des techniques nouvelles déjà bien établies ailleurs et ce, non pas sous forme d'innovations proprement dites ${ }^{2}$. Dans ces pays, les entreprises s'engagent rarement dans la recherche et le développement de nouvelles techniques mais plutôt dans l'adoption et l'adaptation de techniques établies. Par conséquent, si l'on envisage la fonction de production comme la collection de toutes les techniques disponibles et faisables, le remplacement d'une technique par une autre correspondrait à un mouvement le long de la fonction de production, ou à un mouvement de l'intérieur vers la surface de la fonction de production. Puisque, dans ces cas, il n'y a pas de déplacement de la fonction, il n'y aurait donc pas, selon la théorie conventionnelle, de progrès technologique dans les PVD. Cette conclusion est évidemment fausse et remet en question la validité du concept économique de progrès technologique.

Pour remédier à cette difficulté on doit s'attaquer à la définition même de la fonction de production. Dans son important ouvrage sur la productivité et le changement technologique, Salter a discuté deux façons de définir la fonction de production:

«... there are two ranges of alternative techniques and the production function could refer to either. The first is the relatively narrow range of developed techniques actually available to businessmen - the range exemplified by the specifications of machines on the market. The second is the much wider range of techniques which could be designed with the current stock of knowledge. ${ }^{3}$

Bien que Salter ait adopté la dernière des deux définitions il était conscient du caractère "nébuleux» de ce concept ${ }^{4}$.

La fonction de production reflétant l'état actuel des connaissances scientifiques et techniques, que nous allons appeler la fonction de production hypothétique et universelle, est un concept sans doute élégant mais souvent inapproprié. Il permet, notamment dans sa forme néoclassique, de distinguer entre un changement du niveau général des con-

1. Rosenberg (1976), p. 63.

2. Dans un sens plus strict le terme innovation se réfère à un avancement technologique à l'échelle mondiale. Voir par exemple R. Nelson, S. Winter (1977), p. 36.

3. Salter (1966), pp. 14, 15.

4. "On the other hand, the alternative concept of a range of techniques which could be designed with a given fund of knowledge is a relatively nebulous idea for the very process of design of ten adds to the fund of knowledge», ibid., p. 15. 
naissances (déplacement de l'isoquant) et la simple substitution entre facteurs (mouvement le long de l'isoquant) induite par un changement des prix des facteurs. Cependant, de telles fonctions de production hypothétiques et universelles sont en réalité difficilement observables. Les études empiriques de la production sont toujours basées sur un nombre restreint d'observations dans une région quelconque du monde et n'incluent pas toute la gamme des techniques existantes et potentielles.

On peut donc se demander si pour l'estimation des fonctions de production empiriques, il est défendable d'utiliser les mêmes hypothèses et d'adopter la même forme que pour la fonction de production hypothétique et universelle. Tout dépend évidemment de l'utilisation qu'on en fait. Nous sommes intéressés ici à deux types d'études dans lesquelles on utilise des fonctions de production empiriques: les études du progrès et du transfert technologiques, et les études du choix technologique et de ses implications pour l'emploi des facteurs. Dans le premier cas, il s'agit de comparer les relations de production avant et après le changement technologique. Dans le deuxième cas, on voudrait savoir dans quelle mesure les proportions des facteurs varieraient à la suite d'un changement dans l'environnement économique de l'entreprise. Alors que dans le premier type d'études la fonction de production décrit les relations de production existantes au pays, le choix technologique quant à lui doit se faire à partir de toutes les alternatives que l'on peut rencontrer à l'échelle mondiale, comparable à l'éventail de choix d'un menu gastronomique. Nous nous consacrerons donc d'abord au problème du progrès et du transfert technologiques pour ensuite analyser le choix technologique.

\section{II- LE TRAITEMENT DU PROGRESS ET DU TRANSFERT TECHNOLOGIQUES}

Dans l'analyse du progrès et du transfert technologiques on utilise traditionnellement la fonction de production empirique ${ }^{6}$. Elle doit refléter le plus fidèlement possible les coefficients de production réels dans la région d'étude ${ }^{7}$ et elle est estimée à l'aide de données observées. Elle est la frontière de la meilleure performance du passé dans toutes les techniques utilisées.

5. Nous distinguons ici trois types de techniques, a) techniques existantes et déjà utilisées dans la région, b) techniques existantes non utilisées (non transférées), c) techniques potentielles, i.e., celles qui sont possibles sur la base du savoir scientifique et technique disponible, mais qui ne sont pas encore commercialement développées et nécessitent de la R \& D pour la mise au point.

6. Pour le progrès technologique voir par exemple Solow (1957) et pour le transfert technologique Kmenta (1966) ou Hamdani et Mahmoud (1976).

7. Les termes région et pays sont interchangeables dans cette étude. 
La fonction de production empirique ainsi définie se distingue de la fonction hypothétique et universelle sous trois aspects :

(1) elle exclut toutes les techniques potentielles, i.e. les techniques possibles sur la base du savoir scientifique et technologique, mais pas encore développées et donc nécessitant des dépenses de R \& $D$;

(2) elle exclut toutes les techniques qui ne sont pas présentement utilisées dans la région d'étude pour diverses raisons, mais qui sont déjà utilisées ailleurs dans le monde;

(3) les isoquants de cette fonction sont plus éloignés de l'origine que ceux de la fonction universelle à cause de l'efficacité moindre de certains facteurs de production ${ }^{8}$.

En somme, la fonction empirique est contrainte par la disponibilité commerciale des techniques, l'efficacité locale des facteurs de production et par le coût d'information.

Le progrès technologique dans les PVD prend généralement les formes suivantes: l'acquisition de techniques nouvelles mais bien établies ailleurs, l'adaptation de ces techniques aux conditions locales, et la formation des ouvriers et des cadres. La simple acquisition d'une nouvelle technique sous forme d'équipements, d'information technique et de licences n'est pas équivalente à un transfert technologique complet. Il faut aussi que le savoir-faire et l'expérience soient transmis pour qu'une technique soit véritablement transférée. L'amélioration de la qualité du facteur travail, i.e. la formation de capital humain, est un ingrédient essentiel dans le transfert de techniques. Si ces conditions sont remplies, nous pouvons envisager le résultat $\mathrm{du}$ transfert technologique, ou du progrès technologique ainsi obtenu, comme un déplacement de la fonction de production empirique. Dans l'espace bi-dimensionnel ceci est équivalent à un déplacement des isoquants vers l'origine.

Le progrès technologique causé par l'apprentissage, tel qu'analysé par Lundberg sous le terme de "Horndal effect" ${ }^{9}$ et par Arrow comme "learning by doing " ${ }^{\mathbf{1 0}}$ a été invoqué pour justifier un déplacement général de la fonction de production. Cependant, l'apprentissage se fait surtout dans les techniques actuellement utilisées et ne transmet pas nécessairement ses effets sur toutes les techniques disponibles. Si, dans les termes d'Atkinson et Stiglitz, le progrès technologique est «localisé »

8. L'influence des différences internationales d'efficacité du facteur travail sur la position des isoquants est traité dans Siggel (1982).

9. Lundberg (1961), pp. 129-33.

10. Arrow (1962). 
dans des techniques particulières ${ }^{11}$, la fonction de production se déplace dans quelques parties seulement. Cette description du progrès technologique par Atkinson et Stiglitz nous paraît plus utile que le déplacement généralisé de l'isoquant.

Notre redéfinition de la fonction de production empirique a des implications pour sa forme. L'absence des techniques potentielles ainsi que des techniques existantes non transférées implique que la surface de la fonction de production empirique et ses isoquants ne sont pas nécessairement continus à travers toutes les combinaisons de facteurs. Par conséquent, avec une fonction de production empirique incorrectement spécifiée le progrès technologique risque d'être sous-estimé. Pour illustrer cette possibilité, la figure 1 montre les facteurs d'intrants d'une industrie manufacturière tels qu'observés dans un pays peu industrialisé. On n'a utilisé que deux techniques dans le passé, une «basse " technique $(A)$ qui est artisanale, de petite échelle et intensive dans l'utilisation du facteur travail, et une «haute» technique $(B)$ qui est industrielle, fortement mécanisée et intensive en capital. Les isoquants de $A$ et $B$ sont tracés de façon à permettre un certain degré de substitution ex post des facteurs capital et travail, mais ils excluent la substitution ex ante $e^{12}$. Des techniques intermédiaires en tant que degré de mécanisation et intensité factorielle $K / L$ existent, mais n'ont pas été utilisées dans la région d'étude. Admettons aussi que les deux techniques se distinguent par l'échelle à laquelle l'opération devient rentable ainsi que par la nature du produit et des intrants.

La raison principale pour laquelle il est difficile d'accepter une seule fonction néoclassique pour toutes les techniques d'une industrie est la diversité technologique des multiples processus, surtout du point de vue des économies d'échelle. Comme l'avait déjà argumenté GeorgescuRoegen, il est difficile sinon impossible de réunir tous les processus possibles pour la fabrication d'un produit dans une seule fonction de production $^{13}$ : Ceci est d'autant plus vrai au niveau d'une industrie que les données statistiques des industries englobent souvent une multitude de produits. Les techniques modernes et fortement capitalistes sont généralement plus efficaces, à grande échelle, que les techniques traditionnelles. Ces dernières sont souvent inefficaces à grande échelle ${ }^{\mathbf{1 4}}$. L'agrégation des techniques $A$ et $B$ dans la figure 1 résulterait en une fonction de production de l'industrie qui n'aurait pas les caractéristiques habituelles

11. Atkinson, Stiglitz (1969).

12. Pour une discussion de la substitution ex ante et ex post voir Winston (1974).

13. Georgescu-Roegen (1969).

14. L'influence des économies d'échelle a été analysée, par exemple, par Stewart (1977) et Hawrylyshyn (1975), Partie II, pp. 18-24. 
FIGURE 1

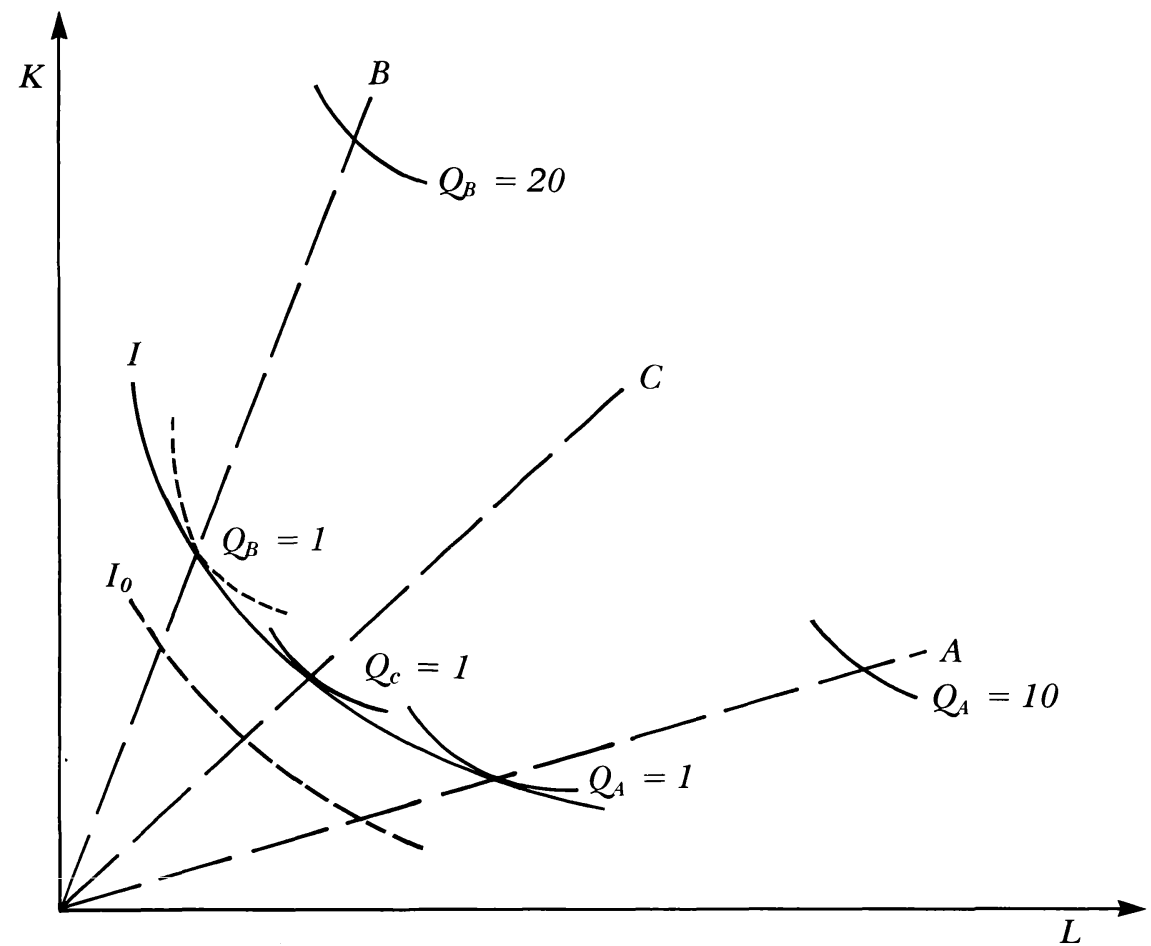

telles que l'homothéticité et des isoquants continus. L'isoquant unitai$\mathrm{re}^{15}$ de $B$ a donc été tracé de façon discontinue pour signaler qu'à ce niveau de production les intrants réels seraient plus élevés.

Supposons que de nouveaux investissements dans cette industrie permettent de transférer une nouvelle technique de type $C$ qui se situe dans l'espace intermédiaire de $A$ et $B$. Si l'on avait tracé un seul isoquant sous forme d'enveloppe (tel que $I$ ) comme image de la technologie avant le transfert, l'introduction de $C$ ne se qualifierait pas nécessairement de progrès technologique. Dans ce cas l'adoption de la technique $C$ ne serait du progrès technologique qu'à condition qu'elle résulte en un déplacement vers l'origine de l'isoquant $I$. Cette condition nous parait trop restrictive. Le problème résulte de la spécification inappropriée de la fonc-

15. L'isoquant unitaire reflète des coefficients moyens de facteurs et non pas les quantités d'intrants nécessaires pour produire une unité. Il est spécifique pour une échelle de production donnée et une échelle à laquelle la production est efficace. 
tion de production empirique. Si les isoquants $Q_{A}$ et $Q_{B}$ décrivent plus fidèlement l'état technologique de l'industrie dans la région d'étude que leur enveloppe $I$, l'introduction de la technique $C$ établit alors un nouvel isoquant $Q_{c}$ ou, alternativement, un déplacement de $Q_{A}$ ou de $Q_{B}$. La technique $C$ peut résulter d'un mélange de $A$ et $B$ au niveau des différentes phases de transformation. La combinaison d'un équipement moderne $(B)$ dans la phase de transformation principale avec un équipement moins sophistiqué $(A)$ dans les opérations auxiliaires est une forme d'adaptation technologique observée par plusieurs auteurs dans les $\mathrm{PVD}^{16}$. Nous considérons ces formes d'innovation comme étant du progrès technologique tant qu'elles permettent de nouvelles combinaisons de facteurs et tant qu'il y a un ensemble de prix des facteurs pour lequel le coût de $C$ est inférieur au coût de $A$ et $B$. Possiblement l'introduction de $C$ laisse toutes les techniques précédentes $(A$ et $B)$ efficaces ${ }^{17}$. Notre refus de l'isoquant $I$ comme reflet de l'état technologique actuel d'un pays est basé sur la distinction entre la substitution ex ante et la substition ex post. La fonction de production empirique étant la réflexion des investissements passés ne permet que la substitution ex post et exclut toute substitution ex ante. Nous verrons dans la prochaine section que la situation est différente en ce qui concerne le choix technologique qui inclut la substitution ex ante.

\section{III- LE CHOIX DES TECHNIQUES ET LE RAYON TECHNOLOGIQUE}

Les arguments de la section précédente, bien qu'ils semblent mettre en question des concepts fondamentaux de la théorie économique, ont pourtant un objectif plus modeste. Il s'agit de critiquer l'usage souvent inapproprié de la fonction de production théorique dans les études empiriques. C'est précisément dans les études du choix technologique, que l'application inappropriée des fonctions de production a les conséquences les plus sérieuses.

L'objectif majeur des études du choix technologique est d'analyser et de mesurer la facilité de substitution entre les facteurs de production. Cette tâche a été accomplie traditionnellement par l'estimation économétrique des fonctions de production. Le calcul de l'élasticité de substitution permet ensuite de déterminer de combien les proportions de facteurs changeraient à la suite d'une variation dans les prix des facteurs ${ }^{18}$. Si la fonction de production utilisée englobe plus que les possibilités réelles de substitution entre facteurs, il est clair que la prévision de l'impact

16. Voir par exemple Pack (1976), Morley et Smith (1974) et Veldhuis (1979).

17. Dans un contexte similaire d'analyse d'activités Hawrylyshyn (1975) a distingué des changements technologiques dominants, partiellement dominants et non dominants. La dernière catégorie est celle qui laisse toutes les techniques précédentes efficaces (p. 199).

18. Pour une revue d'études de ce genre voir Morawetz (1976), pp. 11-15. 
d'une politique affectant les prix des facteurs serait incorrecte. Cette méthode conventionnelle de définir et de mesurer le choix technologique est déficiente selon deux points de vue. D'abord, la méthode présuppose que les choix technologiques se font strictement sur la base de la minimisation des coûts, alors qu'il est bien connu que d'autres facteurs entrent en ligne de compte ${ }^{19}$. Ensuite, et ceci est notre principal argument, l'hypothèse que les fonctions néo-classiques de production estimées sur la base de l'ensemble des données de production représentent bien la gamme réelle des possibilités de substitution, n'est pas toujours défendable au niveau micro-économique.

Quand il s'agit d'examiner les conséquences d'un changement des prix des facteurs sur l'emploi total dans l'économie il est approprié d'utiliser une fonction de production macro-économique. Plus le niveau d'agrégation est élevé, plus la forme néo-classique est justifiable étant donné que les possibilités de substitution se multiplient. La substitution entre facteurs de production devient alors une substitution entre produits et même entre industries, plutôt qu'entre différents processus de production. Les analyses d'équilibre général sont alors plus appropriées que les analyses d'équilibre partiel. Dans la présente étude, nous proposons cependant une approche qui n'est pas seulement microéconomique et d'équilibre partiel, mais qui tient compte également de l'information technique généralement ignorée dans les études économiques.

Dans les études de progrès et de transfert technologiques, on analyse l'ensemble des techniques déjà utilisées dans la région à l'étude. Le choix technologique, par contre, se fait entre les techniques déjà utilisées et celles qui ne le sont pas encore. C'est une perspective ex ante alors que celle du cas précédent était ex post. Il faut souligner cependant que nous excluons toujours les techniques potentielles telles que définies plus haut ${ }^{20}$. Le concept qui convient le mieux pour résumer les possibilités du choix technologique est celui du rayon technologique (technology shelf). Ranis a défini le concept comme suit :

"The technology shelf is composed of the complete set of such activities or technologies which have been demonstrated to be feasible somewhere in the advanced countries at some historical point in time, including the present. „ ${ }^{21}$

Le rayon technologique auquel font face les investisseurs d'un pays ou d'une région est une collection de techniques qui dépassent l'ensemble

19. Voir par exemple Mason (1973), Picket (1974) et Wells (1975). L'argument de Wells est que le choix technologique se fait généralement par les ingénieurs qui ont des objectifs différents de la maximisation des profits.

20. Voir note 5 .

21. Ranis (1973), p. 392. 
des techniques actuellement en usage. Il englobe également toutes les techniques qui ont déjà été appliquées ailleurs et qui sont à la fois faisables et transférables avec les ressources locales. La contrainte de la rareté et de la qualité des ressources locales est importante comme dans l'étude du progrès technologique. Si le rayon technologique correspond à l'isoquant de la fonction de production on peut dire que celui-ci est plus éloigné de l'origine dans les PVD que dans les pays industrialisés à cause des différences dans l'efficacité des facteurs, notamment du travail. De plus, il nous parait possible que certaines techniques soient parfaitement impossibles ou inefficaces par rapport au reste du rayon. C'est pour cela que nous envisageons le rayon technologique d'une région particulière comme un ensemble moins complet que le rayon universel.

Certaines ressources humaines et naturelles peuvent être importées. Cette possibilité élargit l'ensemble des techniques faisables. Cependant, toutes les compétences technologiques ne s'importent pas, surtout à long terme. Ceci est le problème principal des transferts technologiques. L'acquisition des compétences techniques peut être un long processus coûteux qui nécessite l'expérience, la formation, l'éducation et la prise de responsabilités. Le manque de compétences techniques dans un PVD est la raison pour laquelle son rayon technologique diffère du rayon technologique universel basé sur la meilleure performance à l'échelle mondiale (isoquant $I_{0}$ dans la figure 1).

On peut conclure de cette discussion que pour identifier le rayon technologique tel que les investisseurs d'un pays le perçoivent, il ne suffit pas de définir le produit de l'industrie en question mais il faut également spécifier les contraintes liées à la disponibilité et à la qualité des facteurs de production. Puisque ces contraintes changent avec le temps il est nécessaire de définir le rayon technologique en fonction du temps. Cette précision est similaire à la définition du court et du long terme pour la fonction des coûts. Le rayon technologique se définit bien sûr dans le long terme, car le capital est variable. Il exclut le très long terme, cependant, dans lequel les caractéristiques du facteur humain changent aussi.

Pour mesurer les possibilités de substitution d'une industrie particulière, il faut déterminer le rayon technologique auquel fait face l'entreprise au moment d'un investissement. Une approche à ce problème est de questionner directement les entrepreneurs et techniciens afin de pouvoir tenir compte du niveau de formation technique actuellement disponible. L'information désirée doit être en termes de quantité de facteurs requise pour chacune des techniques faisant partie du rayon technologique. Si une telle enquête n'est pas faisable, on peut sur la base des données internationales, estimer les coefficients de toutes les techniques 
jugées disponibles et faisables en tenant compte de la productivité des facteurs dans la région donnée ${ }^{22}$.

Ayant mis en doute que la fonction néoclassique sous forme d'enveloppe reflète adéquatement les fonctions de production empiriques de certaines industries particulières, nous pouvons tout de même nous demander si elle n'est pas une approximation raisonnable du rayon technologique de ces industries. Le fait que le dernier doit refléter un nombre de techniques beaucoup plus grand que l'ensemble des techniques observées dans le passé suggère une réponse positive. Plus précisément, cela dépend des caractéristiques technologiques de l'industrie particulière. Prenons encore une fois le cas d'une industrie décrite à la figure 1 . Si les techniques $A$ et $B$ ne permettent pas de combiner des éléments de $A$ et $B$ pour obtenir $C$ il sera difficile d'accepter l'hypothèse que l'isoquant $I$ reflète fidèlement les possibilités réelles de substitution. Étant donné que des techniques potentielles (qu'on peut toujours invoquer pour la fonction hypothétique et universelle) ne font pas partie du rayon technologique, on peut répondre à cette question en examinant l'inventaire complet des techniques existantes. Dans la prochaine section, nous étudierons des différentes façons de générer de l'information sur les possibilités réelles de substitution à partir de sources d'information diverses, y compris le génie industriel.

En conclusion, la fonction néoclassique ne peut être acceptable comme approximation du rayon technologique d'une industrie dans une région déterminée que si deux conditions sont remplies:

a) un grand nombre de techniques alternatives doivent exister dans l'industrie en question;

b) l'état de développement technologique du pays ou de la région en question doit être suffisamment avancé pour permettre que de nouvelles techniques soient transférables dans la région.

La première condition est une question empirique et varie d'une industrie à l'autre. La deuxième condition implique que dans les pays les moins développés, il est plus difficile de définir le rayon technologique particulier parce que plusieurs techniques du rayon universel sont susceptibles d'être non transférables dans les conditions actuelles. Ceci s'applique même aux techniques artisanales.

\section{IV- L'ÉVALUATION DES POSSIBILITÉS DE SUBSTITUTION: UNE PROPOSITION}

Si la fonction de production néo-classique peut être un instrument inapproprié pour évaluer les possibilités de substitution, il faut alors éta-

22. Une approche semblable a été présentée par Pack (1974), utilisant des données internationales. 
blir d'autres méthodes d'évaluation que l'élasticité de substitution. Une première mesure partielle est l'étendue de substitution qui se définit comme étant l'étendue entre la technique la plus intensive et la moins intensive en termes de capital. Elle peut se représenter, par exemple, sous la forme suivante:

$$
E=\frac{(K / L)_{\max }-(K / L)_{\min }}{(K / L)_{\max }}
$$

où $K / L$ est la proportion des facteurs capital et travail, observables dans une industrie donnée. Cette mesure a été proposée précédemment dans cette revue par Hawrylyshyn, afin d'évaluer certains effets du changement technologique ${ }^{23}$. Elle a été utilisée également par Ferguson ${ }^{24}$ et par d'autres $^{25}$ sous des formes légèrement différentes.

L'étendue de substitution est cependant une mesure incomplète pour expliquer la facilité de substitution, puisqu'il nous faut encore déterminer si à l'intérieur de cette étendue, il existe effectivement de nombreuses techniques ou combinaisons d'éléments de techniques. Si tel est le cas, nous parlerons alors d'une grande densité de substitution. Celle-ci peut se définir simplement comme étant le nombre total de techniques possibles, mais la mesure serait plus significative si le nombre était mis en relation avec l'étendue de substitution. Il est sans doute possible de raffiner ce concept de densité mais, à notre avis, cela n'apporterait pas d'avantages supplémentaires parce que la mesure ne tiendrait toujours pas compte de tous les aspects du choix technologique. Étant donné la complexité de ce choix, le problème ne se résout pas facilement par des méthodes quantitatives.

L'étendue et la densité de substitution sont déterminées par un certain nombre de caractéristiques propres à l'industrie. Parmi ces caractéristiques influençant la facilité de substitution, nous en considérerons seulement quatre : la différenciation du produit, la profondeur de transformation, les économies d'échelle et la facilité de mécanisation. Ces caractéristiques sont universelles en comparaison avec des caractéristiques régionales telles la disponibilité et la productivité des ressources humaines et naturelles. Néanmoins, il peut y avoir une variation régionale parmi les caractéristiques universelles des industries.

\section{Différenciation du produit}

Le degré de différenciation ou d'hétérogénéité du produit est susceptible d'influencer à la fois l'étendue et la densité de substitution. Plus

23. Hawrylyshyn (1977), p. 194.

24. Ferguson (1964).

25. Voir le traitement par Sutcliffe (1971), chapitre 5.2. 
un produit est différencié, plus les processus de fabrication sont variés et plus l'étendue de substitution est large. L'introduction de nouvelles matières premières augmente le nombre de techniques disponibles et le résultat de ces combinaisons est reflété par un haut degré de différenciation du produit. L'influence de cette caractéristique sur le choix technologique varie d'une industrie à l'autre. Il est facile de trouver des produits pour lesquels la différenciation de produits ne semble pas influencer la technique. Par exemple dans l'industrie pharmaceutique, le contenu chimique de certains médicaments n'influence que très peu la technologie de production. Dans d'autres industries, un produit légèrement différent peut exiger un équipement supplémentaire et ainsi augmenter l'étendue et la densité de substitution. Une façon simple de mesurer le degré de différenciation serait par le nombre de produits et de variétés de produits offerts par une industrie.

\section{Profondeur de transformation}

Par la profondeur de transformation, nous entendons ici le nombre de phases distinctes dont se compose le processus de transformation où chacune des phases se définit par une ou plusieurs machines. La notion de profondeur de transformation est reliée au degré d'intégration verticale et s'exprime par divers aspects de l'organisation de la production, par exemple le degré de sous-traitance. L'industrie de l'automobile, par exemple, se présente souvent dans les PVD sous forme d'assemblage CKD ou $\mathrm{SKD}^{26}$. Dans ce cas, nous parlons de transformation peu profonde en comparaison avec les industries d'automobiles des pays industrialisés dont le degré d'intégration verticale est généralement plus grand. Plus une industrie est verticalement intégrée, plus elle offre de possibilités pour combiner des techniques différentes. Par conséquent, ce sont des caractéristiques techniques ainsi que l'organisation industrielle des industries qui influencent la densité de substitution.

\section{Économies d'échelle}

Une industrie caractérisée par de fortes économiē d'échelle risque de produire en dessous du seuil minimum de rentabilité. Des unités de production inférieures à ce niveau minimum d'efficacité sont généralement éliminées par la compétition. S'il y avait des économies d'échelle relatives, c'est-à-dire si le marché national permettait l'existence d'une seule unité de production (le cas du monopole naturel), les petites et moyennes entreprises disparaîtraient complètement à cause de leurs coûts plus élevés. Par conséquent, le nombre de techniques efficaces est

26. Les systèmes d'assemblage d'automobiles CKD (completely knocked down) et SKD (semi knocked down) différent par le nombre d'opérations qu'englobe l'assemblage. 
réduit. Nous pouvons donc conclure que l'étendue et la densité de substitution sont réduites par la présence d'économies d'échelle.

\section{Facilité de mécanisation}

Une autre caractéristique industrielle fondamentale est la facilité avec laquelle un processus de production se mécanise. Certains procédés tels que le raffinage du pétrole et d'autres processus chimiques, se prêtent facilement à la mécanisation et à l'automation. D'autres, tels que la construction de machines ou encore la production de chaussures et de vêtements se mécanisent plus difficilement. Ce dernier type d'industrie est d'ailleurs resté plus imperméable au progrès technologique que le premier. Nous considérons la facilité de mécanisation bien qu'elle soit une variable difficilement quantifiable, comme le principal déterminant de l'intensité en capital. Nous croyons donc qu'une grande facilité de mécanisation est susceptible de réduire l'étendue et la densité de substitution.

\section{V- QUELQUES DONNÉES EMPIRIQUES}

Pour soumettre les considérations précédentes à un test empirique, nous avons évalué l'étendue et la densité de substitution sur la base d'informations recueillies en 1975 et 1976 dans treize industries manufacturières du Zaïre. Les renseignements ont été obtenus par questionnaire et des interviews avec des administrateurs, ingénieurs et techniciens dans 65 entreprises. Au tableau 1 les quatre caractéristiques discutées précédemment sont évaluées en fonction de leur influence positive ou négative sur l'étendue (E) et la densité (D) de substitution. Ces évaluations sont arbitraires et ne reflètent que notre perception des relations étudiées, basée sur les renseignements obtenus. La pondération relative des caractéristiques est spécialement problématique.

La profondeur de transformation (colonne 1) est mesurée par le nombre de sous-processus. Si elle est grande ( $\geqslant 6$ sous-processus) elle renforce la densité $(+D)$ de substitution. La facilité de mécanisation (colonne 2), qui est la moins mesurable des variables, est qualifiée de grande ou faible, selon le cas. Son influence sur l'étendue et la densité de substitution est indiquée par $+\mathrm{E},+\mathrm{D}$ ou $-\mathrm{E},-\mathrm{D}$. Ces deux caractéristiques relèvent fortement du domaine technique et ne semblent pas varier d'un pays à l'autre. Elles ne sont observées qu'au niveau universel. Le reste des caractéristiques ne prennent pas nécessairement les mêmes valeurs au niveau régional qu'au niveau mondial.

Le degré de différenciation du produit (colonnes 3a et 3b) est qualifié soit de fort, renforçant l'étendue $(+E)$ et la densité $(+D)$, soit de faible $(-E$ et $-\mathrm{D})$. Une industrie qui produit, à l'échelle mondiale, beaucoup de produits différents peut être représentée par un seul produit homogène au 
TABLEAU 1

FACTEURS INFLUENÇANT L'ÉTENDUE (E) ET LA DENSITÉ (D) DE SUBSTITUTION DANS TREIZE INDUSTRIES MANUFACTURIÈRES AU ZAÏRE

\begin{tabular}{|c|c|c|c|c|c|c|c|c|c|c|c|c|c|c|}
\hline \multirow[b]{2}{*}{ Industrie } & \multirow{2}{*}{$\begin{array}{l}\text { Profondeur } \\
\text { de } \\
\text { transformation }\end{array}$} & \multirow{2}{*}{$\begin{array}{l}\text { Facilité } \\
\text { de } \\
\text { mécanisation }\end{array}$} & \multicolumn{2}{|c|}{$\begin{array}{l}\text { Degré de } \\
\text { différenciation } \\
\text { du produit }\end{array}$} & \multicolumn{2}{|c|}{$\begin{array}{l}\text { Économies } \\
\text { d'échelle }\end{array}$} & \multicolumn{2}{|c|}{$\begin{array}{l}\text { Existence de PME } \\
\text { ou artisanat }\end{array}$} & \multicolumn{2}{|c|}{$\begin{array}{l}\text { Total : conditions } \\
\text { universelles }\end{array}$} & \multicolumn{2}{|c|}{$\begin{array}{l}\text { Total: conditions } \\
\text { Zaire }\end{array}$} & \multirow{2}{*}{$\begin{array}{l}\begin{array}{l}\text { Etendue } \\
\text { observée } \\
(K / L) \max \end{array} \\
(K / L) \min \end{array}$} & \multirow{2}{*}{$\begin{array}{l}\text { Nombre } \\
\text { d'établis- } \\
\text { sements } \\
\text { étudiés }\end{array}$} \\
\hline & & & $\begin{array}{l}\text { niveau } \\
\text { universel }\end{array}$ & Zaïre & $\begin{array}{l}\text { niveau } \\
\text { universel }\end{array}$ & Zaïre & $\begin{array}{l}\text { niveau } \\
\text { universel }\end{array}$ & Zaïre & Étendue & Densité & Étendue & Densité & & \\
\hline & (1) & (2) & (3a) & (3b) & (4a) & (4b) & (5a) & (5b) & (6) & (7) & (8) & (9) & (10) & (11) \\
\hline \multicolumn{15}{|c|}{ A. Industries de type "processus" } \\
\hline Boulangeries & $\begin{array}{l}9 \\
+\mathrm{D} \\
\end{array}$ & $\begin{array}{l}\text { grande } \\
-E-D\end{array}$ & $\begin{array}{l}\text { haut } \\
+\mathrm{E}+\mathrm{D}\end{array}$ & $\begin{array}{l}\text { bas } \\
-\mathrm{E}-\mathrm{D}\end{array}$ & $\begin{array}{l}\text { n.i. }{ }^{1} \\
+\mathrm{E}\end{array}$ & $\begin{array}{l}\text { i. }^{2} \\
-\mathrm{E}\end{array}$ & $\begin{array}{l}\text { oui } \\
+\mathrm{E}+\mathrm{D}\end{array}$ & $\begin{array}{l}\text { oui } \\
+\mathrm{E}+\mathrm{D}\end{array}$ & $\mathrm{E}=2$ & $\mathrm{D}=2$ & $E=-2$ & $\mathrm{D}=0$ & 15,0 & 8 \\
\hline Sucre & $\begin{array}{l}12 \\
+\mathrm{D} \\
\end{array}$ & $\begin{array}{l}\text { grande } \\
-E-D\end{array}$ & $\begin{array}{l}\text { bas } \\
-\mathrm{E}-\mathrm{D}\end{array}$ & $\begin{array}{l}\text { bas } \\
-\mathrm{E}-\mathrm{D}\end{array}$ & $\begin{array}{l}\text { i. } \\
-E\end{array}$ & $\begin{array}{l}\text { i. } \\
-E\end{array}$ & $\begin{array}{l}\text { oui } \\
+\mathrm{E}+\mathrm{D}\end{array}$ & $\begin{array}{l}\text { non } \\
-E-D\end{array}$ & $E=-2$ & $\mathrm{D}=0$ & $\mathrm{E}=-4$ & $\mathrm{D}=-2$ & 3,7 & 3 \\
\hline Bière & $\begin{array}{l}8 \\
+\mathrm{D} \\
\end{array}$ & $\begin{array}{l}\text { grande } \\
-E-D\end{array}$ & $\begin{array}{l}\text { bas } \\
-\mathrm{E}-\mathrm{D}\end{array}$ & $\begin{array}{l}\text { bas } \\
-\mathrm{E}-\mathrm{D}\end{array}$ & $\begin{array}{l}\text { n.i. } \\
+\mathrm{E}\end{array}$ & $\begin{array}{l}\text { i. } \\
-E\end{array}$ & $\begin{array}{l}\text { non } \\
-E-D\end{array}$ & $\begin{array}{l}\text { non } \\
-\mathrm{E}-\mathrm{D}\end{array}$ & $E=-2$ & $\mathrm{D}=-2$ & $E=-4$ & $\mathrm{D}=-2$ & 2,1 & 2 \\
\hline Boissons douces & $\begin{array}{l}5 \\
-\mathrm{D} \\
\end{array}$ & $\begin{array}{l}\text { grande } \\
-\mathrm{E}-\mathrm{D}\end{array}$ & $\begin{array}{l}\text { bas } \\
-\mathrm{E}-\mathrm{D}\end{array}$ & $\begin{array}{l}\text { bas } \\
-\mathrm{E}-\mathrm{D}\end{array}$ & $\begin{array}{l}\text { n.i. } \\
+\mathrm{E}\end{array}$ & $\begin{array}{l}\text { i. } \\
-E\end{array}$ & $\begin{array}{l}\text { oui } \\
+\mathrm{E}+\mathrm{D}\end{array}$ & $\begin{array}{l}\text { non } \\
-E-D\end{array}$ & $E=0$ & $\mathrm{D}=-2$ & $E=-4$ & $\mathrm{D}=-4$ & 1,4 & 4 \\
\hline Cigarettes & $\begin{array}{l}8 \\
+\mathrm{D}\end{array}$ & $\begin{array}{l}\text { grande } \\
-E-D\end{array}$ & $\begin{array}{l}\text { bas } \\
-\mathrm{E}-\mathrm{D}\end{array}$ & $\begin{array}{l}\text { bas } \\
-\mathrm{E}-\mathrm{D}\end{array}$ & $\begin{array}{l}\text { i. } \\
-E\end{array}$ & $\begin{array}{l}\text { i. } \\
-E\end{array}$ & $\begin{array}{l}\text { oui } \\
+\mathrm{E}+\mathrm{D}\end{array}$ & $\begin{array}{l}\text { non } \\
-E-D\end{array}$ & $E=-2$ & $\mathrm{D}=0$ & $\mathrm{E}=-4$ & $\mathrm{D}=-2$ & 1,3 & 3 \\
\hline Tissus textiles & $\begin{array}{l}11 \\
+\mathrm{D} \\
\end{array}$ & $\begin{array}{l}\text { grande } \\
-E-D\end{array}$ & $\begin{array}{l}\text { haut } \\
+\mathrm{E}+\mathrm{D}\end{array}$ & $\begin{array}{l}\text { haut } \\
+\mathrm{E}+\mathrm{D}\end{array}$ & $\begin{array}{l}\text { n.i. } \\
+\mathrm{E}\end{array}$ & $\begin{array}{l}\text { n.i. } \\
+E\end{array}$ & $\begin{array}{l}\text { oui } \\
+\mathrm{E}+\mathrm{D}\end{array}$ & $\begin{array}{l}\text { non } \\
-E-D\end{array}$ & $\mathrm{E}=2$ & $\mathrm{D}=2$ & $\mathrm{E}=0$ & $\mathrm{D}=0$ & 3,7 & 6 \\
\hline Savon & $\begin{array}{l}5 \\
-D\end{array}$ & $\begin{array}{l}\text { grande } \\
-E-D\end{array}$ & $\begin{array}{l}\text { bas } \\
-\mathrm{E}-\mathrm{D}\end{array}$ & $\begin{array}{l}\text { bas } \\
-\mathrm{E}-\mathrm{D}\end{array}$ & $\begin{array}{l}\text { n.i. } \\
+\mathrm{E}\end{array}$ & $\begin{array}{l}\text { i. } \\
-E\end{array}$ & $\begin{array}{l}\text { oui } \\
+\mathrm{E}+\mathrm{D}\end{array}$ & $\begin{array}{l}\text { oui } \\
+\mathrm{E}+\mathrm{D}\end{array}$ & $E=0$ & $\mathrm{D}=-2$ & $E=-2$ & $\mathrm{D}=-2$ & 2,0 & 5 \\
\hline \multirow[t]{2}{*}{ Ciment } & $\begin{array}{l}5 \\
-D\end{array}$ & $\begin{array}{l}\text { grande } \\
-E-D\end{array}$ & $\begin{array}{l}\text { bas } \\
-\mathrm{E}-\mathrm{D}\end{array}$ & $\begin{array}{l}\text { bas } \\
-\mathrm{E}-\mathrm{D}\end{array}$ & ${ }_{-E}$ & $\begin{array}{l}\text { i. } \\
-E\end{array}$ & $\begin{array}{l}\text { non } \\
-E-D\end{array}$ & $\begin{array}{l}\text { non } \\
-E-D\end{array}$ & $E=-4$ & $\mathrm{D}=-4$ & $E=-4$ & $\mathrm{D}=-4$ & 3,1 & 3 \\
\hline & & & & & & & moyenne: & & $E=-3 / 4$ & $D=-3 / 4$ & $E=-3$ & $\mathrm{D}=-2$ & & \\
\hline
\end{tabular}

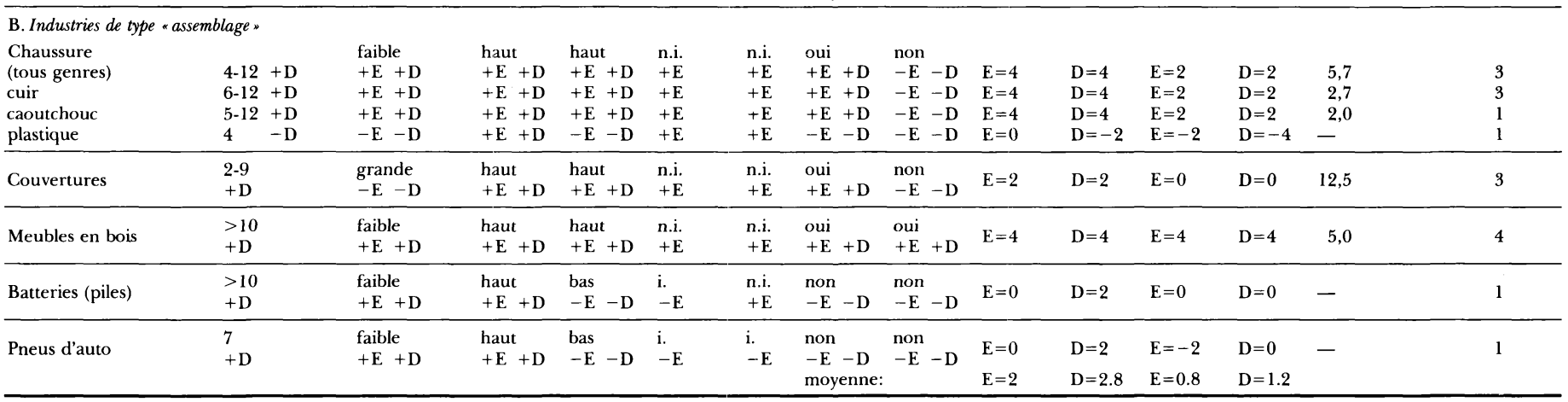

SOIRCF. Interviews dans 46 établissements manufacturiers au Zaïre. 
pays d'étude. Les économies d'échelle (colonnes 4a et 4b) peuvent être importantes en valeur absolue mais peu importantes par rapport à la taille du marché local. L'existence de petites et moyennes entreprises (PME) ou d'un artisanat (colonnes 5a et 5b) est interprétée comme influence positive sur l'étendue et la densité de substitution $(+\mathrm{E},+\mathrm{D})$.

Ayant l'intention de résumer l'influence de ces facteurs, nous avons additionné horizontalement les $\mathrm{E}$ et les $\mathrm{D}$ afin d'obtenir une mesure de l'étendue. Elle est généralement petite (la valeur moyenne de $\mathrm{E}$ étant $-0,75)$ dans les industries de type " processus », et large $(\mathrm{E}$ moyen $=+0,8)$ dans les industries de type "assemblage». La densité de substitution est également inférieure dans les industries de type « processus » (D moyen = $-0,75)$ que dans les industries de type «assemblage» $(\mathrm{D}$ moyen $=+2,8)$. Ces valeurs réfèrent aux conditions universelles. Dans les colonnes suivantes, nous avons répété la même évaluation au niveau régional en tenant compte des caractéristiques locales. Ces facteurs ne sont pas discutés ici parce qu'ils demanderaient des explications substantielles à l'égard des conditions de production industrielle au Zaïre ${ }^{27}$. Comme le tableau 1 le montre, les valeurs de $\mathrm{E}$ et $\mathrm{D}$ sont toujours inférieures au niveau régional qu'au niveau universel.

Nos évaluations de l'étendue et de la densité de substitution sont comparées au tableau 2 aux estimations de l'élasticité de substitution dans 13 industries manufacturières, également au Zaïre, par Cleaver ${ }^{28}$ qui utilisait une fonction de production de type CES. La comparaison est intéressante parce que les données utilisées par Cleaver viennent en grande partie des mêmes firmes que nous avons étudiées en détail, et elles se réfèrent à la même période (1970-74, variant selon le cas). Les observations du tableau 1 représentent la production en 1972, 1973 ou 1974. Suivant le raisonnement antérieur on pourrait s'attendre à une corrélation positive entre la somme de $\mathrm{E}$ et $\mathrm{D}$ et l'élasticité de substitution si ces variables étaient correctement évaluées. Malheureusement il n'était pas possible de calculer un coefficient de corrélation parce que les échantillons ne correspondent pas suffisamment. Comme on peut le constater dans le tableau 2, il n'y a pas de relation apparente entre les valeurs de E et $\mathrm{D}$ (niveau régional) et l'élasticité de substitution. Le seul cas où nos observations sont comparables avec les estimés de Cleaver est l'industrie de caoutchouc qui consiste en deux firmes produisant des chaussures et des pneus de bicyclettes, respectivement. C'est une industrie apparemment flexible technologiquement. Un autre cas intéressant est l'industrie des produits métalliques. C'est l'industrie la plus diversifiée selon ses produits, qui comprennent des meubles métalliques, des ustensiles de cuisine, des constructions métalliques, des bouchons de couronne, des câbles, des tôles et divers

27. Voir Siggel (1978), chapitre 4.

28. K.M. Cleaver (1975), chapitre 2. 
TABLEAU 2

ÉTENDUE, DENSITÉ ET ÉLASTICITÉ DE SUBSTITUTION DANS 17 INDUSTRIES MANUFACTURIËRES

AU ZAÏRE ET COMPARAISON AVEC D’AUTRES ESTIMATIONS

\begin{tabular}{|c|c|c|c|c|c|}
\hline Industrie & $\begin{array}{l}\text { Étendue de } \\
\text { substitution }\end{array}$ & Densité & $\begin{array}{l}\text { Élasticité de } \\
\text { substitution }\end{array}$ & $\frac{(K / L) \max }{(K / L) \min }$ & $\frac{(K / L) \max }{(K / L) \min }$ \\
\hline & (a) & (b) & (c) & (d) & (e) \\
\hline Boulangeries & -2 & 0 & 0,66 & $(18)^{3}$ & $*$ \\
\hline Sucre & -4 & -2 & $*$ & \}$(1,8)^{\circ}$ & 10,3 \\
\hline \multicolumn{6}{|l|}{ Boissons: } \\
\hline Bière & -4 & -27 & & * & 3,0 \\
\hline Douces & -4 & -4 & & $*$ & $*$ \\
\hline Cigarettes & -4 & -2 & 0,62 & 10,85 & 6,2 \\
\hline Tissus textiles & 0 & 0 & 0,62 & 2,6 & 4,8 \\
\hline Savon & -2 & -2 & 0,76 & $(2,1)^{1}$ & $*$ \\
\hline Ciment & $-\overline{4}$ & $-\overline{4}$ & 0,63 & $(2,1)^{2}$ & $*$ \\
\hline Chaussure & 2 & 2 & & $(3,2)^{4}$ & $*$ \\
\hline Cuir & 2 & 2 & & $*$ & * \\
\hline Plastique & $-\overline{2}$ & $-\overline{4}$ & 1,26 & * & $*$ \\
\hline Caoutchouc & 2 & 2 & & * & * \\
\hline \multicolumn{6}{|l|}{ Pneus: } \\
\hline Bicyclettes & $*$ & $*$ & & $*$ & $*$ \\
\hline Automobiles & -2 & 0 & & 3,2 & 2,1 \\
\hline Couvertures & 0 & 0 & $*$ & $*$ & $*$ \\
\hline Meubles en bois & 4 & 4 & 0,29 & * & $*$ \\
\hline Batteries électriques & 0 & 0 & 0,12 & $*$ & $*$ \\
\hline Produits métalliques & $*$ & $*$ & 0,09 & $*$ & $*$ \\
\hline Matériel de transport & * & * & 0,48 & * & * \\
\hline Huile végétale & * & * & 0,61 & * & * \\
\hline Impression & $*$ & $*$ & 0,63 & $*$ & $*$ \\
\hline Bois & $*$ & * & 0,91 & $*$ & $*$ \\
\hline
\end{tabular}

NOTES: * données non disponibles

1. pour le groupe de produits chimiques

2. pour le groupe des produits minéraux non métalliques

3 . pour le groupe des produits alimentaires

4. pour le groupe des vêtements

SOURCES: Colonnes (a) et (b): Tableau 1; Colonne (c): K. Cleaver (1975, p. 230); Colonne (d): C. Ferguson, "Capital Labour Substitution» dans Greenhut \& Whitman (eds.), Essays in Southern Economic Development; Colonne (e): Table 6, UN Bulletin of Industrialization and Productivity, No. 1, p. 27. Les chiffres de ces deux études sont reproduits dans R.B. Sutcliffe (1971), pp. 149-155. 
autres produits. Pour une telle industrie il est difficile d'envisager une fonction de production à coefficients fixes parce qu'elle englobe une multitude de techniques. L'estimé de l'élasticité de substitution de presque zéro selon Cleaver est difficilement réconciliable avec les faits observés dans notre étude.

Une si faible estimation peut résulter du fait, par exemple, que dans plusieurs productions différentes il existe des proportions de facteurs semblables mais des prix différents de facteurs. Une telle coïncidence ne justifie pas, cependant, l'hypothèse d'une élasticité de substitution près de zéro.

Les élasticités estimées par Cleaver dans les industries de meubles, de batteries électriques et du matériel de transport nous paraissent également trop basses. Par contre, dans les industries de type processus où nous avons observé des techniques relativement uniformes entre les entreprises, les estimations de Cleaver sont relativement élevées.

La comparaison de nos résultats avec ceux de Cleaver ne permet pas de conclusions définitives sur les possibilités de substitution dans les industries étudiées. Par contre, elle fournit des exemples pour nos arguments. Il convient de souligner que les évaluations proposées ici sont d'une valeur limitée et ne sont pas supposées remplacer l'estimation conventionnelle des fonctions de production. Mais il nous paraît justifié de conclure que l'estimation de fonctions de production empiriques en vue d'évaluer le choix et le changement technologiques peut facilement conduire à de fausses conclusions et qu'elle devrait être complétée par des analyses sur la nature exacte des techniques en question.

Les résultats de notre évaluation qualitative sont comparés, au tableau 1, à une série de valeurs de l'étendue de substitution (E) calculées sur la base de coefficients de capital par travailleur employé dans les entreprises de notre étude au Zaïre. Les valeurs de la colonne (10) représentent le quotient $(K / L)$ max sur $(K / L)$ min et le capital est exprimé par sa valeur de remplacement. Il existe une légère corrélation entre ces valeurs (colonne 10) et les valeurs de l'étendue E (colonne 8). Le coefficient de corrélation est de 0,36. Nous avons aussi comparé, au tableau 2, les valeurs de $\mathrm{E}_{\text {Zaire }}$ avec les estimations de l'étendue par d'autres études présentées par Sutcliffe ${ }^{29}$. Cette comparaison n'est pas concluante, cependant, à cause de différences dans la définition des industries ainsi que des différences au niveau de l'échantillon ${ }^{30}$.

29. Voir Sutcliffe (1971), pp. 149-155.

30. Les chiffres de Ferguson se réfèrent aux États-Unis en 1958 et l'échantillon des Nations Unies couvre quatre pays en 1945-50: É.U., Mexique, Colombie, Inde. 
CONCLUSION

L'objectif de cette étude est double. D’abord elle veut démontrer que l'estimation de fonctions néoclassiques de production sur la base de données régionales n'est pas toujours appropriée pour l'étude du progrès et du choix technologiques. Pour l'analyse du progrès technologique on devrait utiliser une fonction qui reflète fidèlement les techniques réellement utilisées. Dans ce cas, la fonction néoclassique qui enveloppe toutes les techniques particulières peut facilement surévaluer l'état technologique d'une industrie, ce qui implique une sous-évaluation possible du progrès technologique. Dans le cas du choix technologique la fonction appropriée n'est pas la fonction empirique mais une fonction qui reflète le "rayon technologique». Cette fonction risque de mal évaluer les possibilités de substitution réelles si elle est estimée à l'aide de la fonction néoclassique (enveloppe) sur la base de données de production locales. Nous avons proposé de ne pas traiter une industrie comme une boîte noire, mais d'utiliser toute information disponible y compris l'information du génie industriel. Les renseignements peuvent être rassemblés pour décrire deux caractéristiques du rayon technologique, son étendue et sa densité de substitution.

Les concepts de l'étendue et de la densité de substitution décrivent ensemble le potentiel de substitution sans toutefois être basés sur une fonction mathématique rigoureuse et contraignante. Il ne s'agit pas de rejeter le concept de l'élasticité de substitution là où il peut être appliqué sans trop grand risque. Il s'agit plutôt d'utiliser des renseignements supplémentaires dans les cas où l'estimation conventionnelle de fonctions de production ne semble pas justifiée à cause des circonstances typiques pour les PVD, telles que la petite taille des marchés, des conditions noncompétitives de production, l'absence d'information, la faible efficacité de certains facteurs de production et la segmentation dualiste des marchés des facteurs. A notre avis. il est inapproprié de prétendre qu'une industrie soit caractérisée par une certaine élasticité de substitution simplement parce que les données d'un échantillon s'adaptent bien à la forme choisie d'une fonction de production. Une question préalable est importante: Est-ce que l'ensemble des informations disponibles justifie le choix de cet outil d'analyse?

Eckhard SIGGEL

Département d'économique Université Concordia 


\section{BIBLIOGRAPHIE}

Arrow, K.J. (1962), «The Economic Implication of Learning by Doing», Review of Economic Studies, vol. XXIX (juin).

Atkinson, A.B., Stiglitz, J.B. (1969), «A New View of Technological Change", Economic Journal, vol. LXXIX (septembre).

Cleaver, K.M., "The Effective Rate of Subsidy and Resource Allocation in Zaïre », unpublished Ph.D. thesis, Fletcher School of Law and Diplomacy, 1975.

Ferguson, C. (1964), "Capital-Labour Substitution » in Greenhut et Whitman (éd.), Essays in Southern Economic Development, Durham (N. Carolina).

Georgescu-Roegen, N. (1969), "Process in Farming versus Process in Manufacturing: A Problem of Balanced Development ", in U. Papi et Ch. Nunn (éd.), Economic Problems of Agriculture in Industrial Societies, IEA Conference (Rome), MacMillan.

Hamdani, K.H., Mahmoud, M.A. (1976), "Analysis of the Technology Transfer Cost», The Pakistan Development Review, vol. XV, $\mathrm{n}^{0} 2$.

Hawrylyshyn, O. (1975), The Causes of Underemployment in Developing Countries, Discussion Papers No. 173, 174, 175, Institute for Economic Research, Queens University.

Hawrylyshyn, O. (1977), «La nature de progrès technique et la substitution des facteurs dans les pays en voie de développement », L'Actualité Économique, $53^{\mathrm{e}}$ année, $\mathrm{n}^{\mathrm{o}} 2$.

KmentA, J. (1966), «Economic Theory and the Transfer of Technology , in Spencer, Woroniak (éd.), The Transfer of Technology to Developing Countries, Praeger.

Lundberg, E. (1961), Productivitet öch Rantabilitet (Stockholm: Norstedt and Söner).

Mason, R.H. (1973), «Some Observations on the Choice of Technology by Multinational Firms in Developing Countries ", Review of Economics and Statistics, août.

Morawetz, D. (1976), «Elasticities of Substitution in Industry: What Do We Learn from Econometric Estimates?», World Development, vol. 4, $n^{0} 1$.

Morley, S.A., Smith, G.W. (1975), The Choice of Technology: Multinational Firms in Brazil, Discussion Paper No. 58, Rice University.

Nelson, R.R., Winter, S.G. (1977), «In Search of Useful Theory of Innovation ", Research Policy, 6. 
Pack, H. (1974), "The Employment-Output Trade-off in LDCs: A Microeconomic Approach», Oxford Economic Papers, vol. 26 (novembre).

Pack, H. (1976), "The Substitution of Labour for Capital in Kenyan Manufacturing", The Economic journal, n 86 (mars), pp. 45-58.

Picket, J.D. et al. (1974), "The Choice of Technology, Economic Efficiency and Employment in Developing Countries », World Development, vol. 2 (mars).

RANis, G. (1973), «Industrial Sector Labour Absorption», Economic Development and Cultural Change, vol. 21 (avril).

Rosenberg, N. (1976), Perspectives on Technology, Cambridge University Press.

Salter, W.E.G. (1966), Productivity and Technical Change, Cambridge University Press.

Siggel, E. (1978), Technology Transfer and the Choice of Industrial Technologies in a Developing Country: The Case of Zaïre, Ph.D. Thesis, University of Toronto.

Siggel, E. (1982), "Labour Efficiency and The Choice of Technique in Less Developed Countries" (mimeo).

Solow, R. (1957), "Technical Change and The Aggregate Production Function", Review of Economics and Statistics (août), pp. 312-20.

Stewart, F. (1977), Technology and Underdevelopment, MacMillan Press.

SutCliffe, R.G. (1971), Industry and Underdevelopment, Addison-Wesley.

Veldhuis, K.H. (1979), «Transfer and Adaptation of Technology: Unilever as a Case Study", in A. Robinson (éd.), Appropriate Technologies for Third World Development, IEA Conference (Teheran), MacMillan.

Wells, C. (1975), «Economic Man and Engineering Man: Choice of Technology in a Low-Wage Country ", in C. Timmer et al., (éd.), The Choice of Technology in Developing Countries, Harvard University.

Winston, G.C. (1974), "Factor Substitution Ex Ante and Ex Post», Journal of Development Economics (juin). 